\title{
Large-scale comparison between buoy and SSM/I drift and deformation in the Eurasian Basin during winter 1992-1993
}

\author{
Cathleen A. Geiger ${ }^{1}$ \\ University Space Research Association, Seabrook, Maryland \\ Yunhe Zhao \\ Caelum Research Corporation, Rockville, Maryland \\ Antony K. Liu and Sirpa Häkkinen \\ NASA/Goddard Space Flight Center, Greenbelt, Maryland
}

\begin{abstract}
A method for comparing sea ice velocity, divergence, and shear at the largescale between buoys and Special Sensor Microwave Imager (SSM/I) is presented. For initial testing, the method is applied in the Eurasian Basin because of its relatively simple circulation dominated by the wind. Using eight Argos buoys, 11 strain rate arrays $100-600 \mathrm{~km}$ in size are constructed. Daily $100 \mathrm{~km}$ resolution sea ice motion derived from SSM/I $85 \mathrm{GHz}$ brightness temperatures is sampled $100-1000 \mathrm{~km}$ from the center of the buoy arrays. Over this range of possible scales, a minimum RMS difference (RMSD) for deformation is used to identify an optimal inclusion radius of $600 \mathrm{~km}$ corresponding to a length scale of $1000 \mathrm{~km}$. This length scale is typical of local storms confirming a strong connection between wind and observed sea ice motion. On the basis of all 11 arrays, an average RMSD of $2.48 \pm 0.05$ $\mathrm{cm} \mathrm{s}^{-1}$ for velocity vector and $8.8 \pm 0.9 \times 10^{-8} \mathrm{~s}^{-1}$ using all four deformation components $\left(\partial u_{i} / \partial x_{\jmath}\right)$ is found at the optimal inclusion radius corresponding to average correlation coefficients of $0.896 \pm 0.002$ and $0.729 \pm 0.030$, respectively. RMSD are found to scale with the temporal and spatial uncertainties of the SSM/I suggesting that even better results can be achieved with higher resolution instruments.
\end{abstract}

\section{Introduction}

The development of a wavelet technique by Liu and Cavalieri [1998] and cross-correlation techniques such as those described by Fily and Rothrock [1987], Kwok et al. [1990], and Emery et al. [1991], provide a means to extract high spatial resolution sea ice motion. These new sea ice motion fields hold great promise as input into numerical models and toward understanding sea ice processes. Results, for example, from Drinkwater [1998a,b], Kwok et al. [1998], and Li et al. [1998] have demonstrated the use of satellite-derived sea ice motion to compute snapshot examples of deformation fields. Work by Stern et al. [1995] includes a detailed study of satellite-derived open water production and numerical model formulations based on these results. Liu and

\footnotetext{
${ }^{1}$ Now at Center for Climatic Research, University of Delawa Newark.

Copyright 2000 by the American Geophysical Union.

Paper number 1999JC900285.

0148-0227/00/1999JC900285\$09.00
}

Cavalieri [1998], Liu et al., [1998], and Liu and Zhao, [1998], use a wavelet technique to produce velocity fields and make detailed comparisons against buoys.

Currently, the only reliable way of validating satellitederived sea ice motion is through comparison to in situ buoy measurements. In each of the works cited above, satellite-derived sea ice velocity has been checked against the Argos network of buoys in the Arctic and Antarctic. However, the task of validating deformation is more difficult. Much of the classic work related to in situ sea ice motion using buoy data is analyzed relative to a Lagrangian reference [e.g., Thorndike, 1986; Hibler et al., 1974], while satellite and more recent buoy products are often presented in an Eulerian reference. Velocity as a point displacement per unit time can be calculated from either satellite or buoy in either reference frame at any scale. Deformation, however, is a measure of velocity spatial variability at a specific temporal and spatial scale such that the differences between drifting and gridded frames of reference and the spatial and temporal distribution of these measurements become very important. To first order, the velocity describes the motion of the ice, but it is the deformation 
and associated processes which lead to the creation of open water which in turn affects the formation of new ice and its associated thermodynamics and contributes to the total mass balance. Hence geophysically, the spatial variability of the ice motion and its changes are at least as important as the general circulation of the ice.

To illustrate some of the difficulties involved, consider the Defense Meteorological Satellite Program's (DMSP) Special Sensor Microwave Imager (SSM/I) instrument which has a typical orbit time of $20 \mathrm{~min}$ but a revisit time (i.e., time it takes for the satellite footprint to revisit nearly the same point on the planet) of the order of 1 day. Daily gridded maps of SSM/I brightness temperatures are created via a composite of swaths collected throughout each day and thus contain temporal uncertainties up to scales of 1 day which cannot be ignored. Thus minimum timescales of 3-4 days must be used to produce motion products of reasonable certainty from SSM/I daily maps. Furthermore, because of the grid resolution and geolocation errors for any instrument, there are corresponding spatial uncertainties which must be accounted for. Consider next the validation of such a data set with buoys which are tracked for example via Argos as Lagrangian point measurements with relatively high temporal resolution (about every 3 hours in polar regions), but coverage that is spatially limited as each instrument must be deployed by a manned crew and is subject to environmental hazards. In both cases, there are instrumental limitations which prevent a complete picture of the true spatial variability of the ice. Because of these limitations, the best one can hope for is a consensus between two independent measurements to obtain an estimate of the true state of the ice.

A method which compares both velocity and deformation between satellite-derived motion and buoy data provides a useful means for testing instrument capability and accuracy in processing techniques. Additionally, a method for preprocessing buoy and satellite-derived velocity fields for use in numerical models is desired in a form with minimal temporal and spatial uncertainties but as much of the natural variability as possible. In this paper we examine such possibilities by focusing on the following problem: At what spatial and temporal scale could buoy and satellite data be processed such that the resulting motion products are mutually compatible and sufficient for examining large-scale geophysical sea ice processes? A case study in the Eurasian Basin based on eight buoys from the International Arctic Buoy Program (IABP) database and motion vectors derived via a wavelet method from SSM/I brightness temperatures [Liu and Cavalieri, 1998] are used to address this question.

We begin in section 2 with a description of the data. Then, applying a method traditionally used for buoy strain rate analysis, we examine results from a comparison between buoys and SSM/I for both velocity and deformation. Finally, we make use of these results to identify instrumental limitations and potential applicability of this method to numerical model preprocessing and sea ice process studies. The impact of these results on open water formation processes is left for future studies.

\section{Data}

In this section, we describe the initial processing of the SSM/I and buoy data, identify the region of study, and detail some of the temporal and spatial uncertainties in the selected data sets.

\subsection{SSM/I Preprocessing}

Using DMSP's SSM/I $85 \mathrm{GHz}$ (12.5 km resolution) daily fields from October 1992 to March 1993, sea ice motion for the Arctic region is computed via a twodimensional (2-D) Mexican hat wavelet transform [Liu and Cavalieri, 1998]. To produce these maps, daily composites from SSM/I brightness temperatures are linearly interpolated from the SSM/I grid onto images in a grid with $6.25 \times 6.25 \mathrm{~km}$ pixel size. Then a $2-\mathrm{D}$ Mexican hat wavelet transform is applied to interpolated $\mathrm{SSM} / \mathrm{I}$ images at three scales equal to $2.0,2.42$, and 2.828 times the pixel resolution to isolate features of the order of $25-50 \mathrm{~km}$. The effect of this wavelet transform is essentially a spatial band-pass filter, set with a threshold limit to detect features of a ciosen physical scale in the ice signature. Once the features are located for each day, localized template matching (e.g., $10 \mathrm{pix}$ els) is used to identify (through minimization) similar band-passed features between two chosen scenes [Liu et al., 1999]. For the Arctic, daily scenes are available, but the template matching and resultant displacements are based on scenes that are $\mathbf{4}$ days apart. We subsequently refer to this as a 4-day sliding window, whereby the motion for a given day is based on scenes 2 days prior to and 2 days following the chosen time. The ice displacement vectors so obtained are then divided by the respective time interval to obtain velocity vectors. The resultant velocities from each scale are block-averaged onto a $100 \times 100 \mathrm{~km}$ resolution grid constructed from a polar stereographic projection centered at the north pole with a geographic distortion of zero at $70^{\circ} \mathrm{N}$ latitude and the $x$ axis directed along $45^{\circ} \mathrm{E}$ longitude (i.e., a variation of the standard SSM/I grid). An example of the SSM/I velocity field is shown in Figure 1a.

\subsection{Buoy Preprocessing}

Buoy data at 12-hour intervals are retrieved for the Arctic from October 1992 to March 1993 from the International Arctic Buoy Program database via the web (ftp://iabp.apl.washington.edu/pub/IABP/Documentation.txt). The latitude and longitude longitude positions are transformed to distances in kilometers from the North Pole via the SSM/I polar stereographic projection just referred to. Velocities are computed via distance displacements of the positions divided by the time 


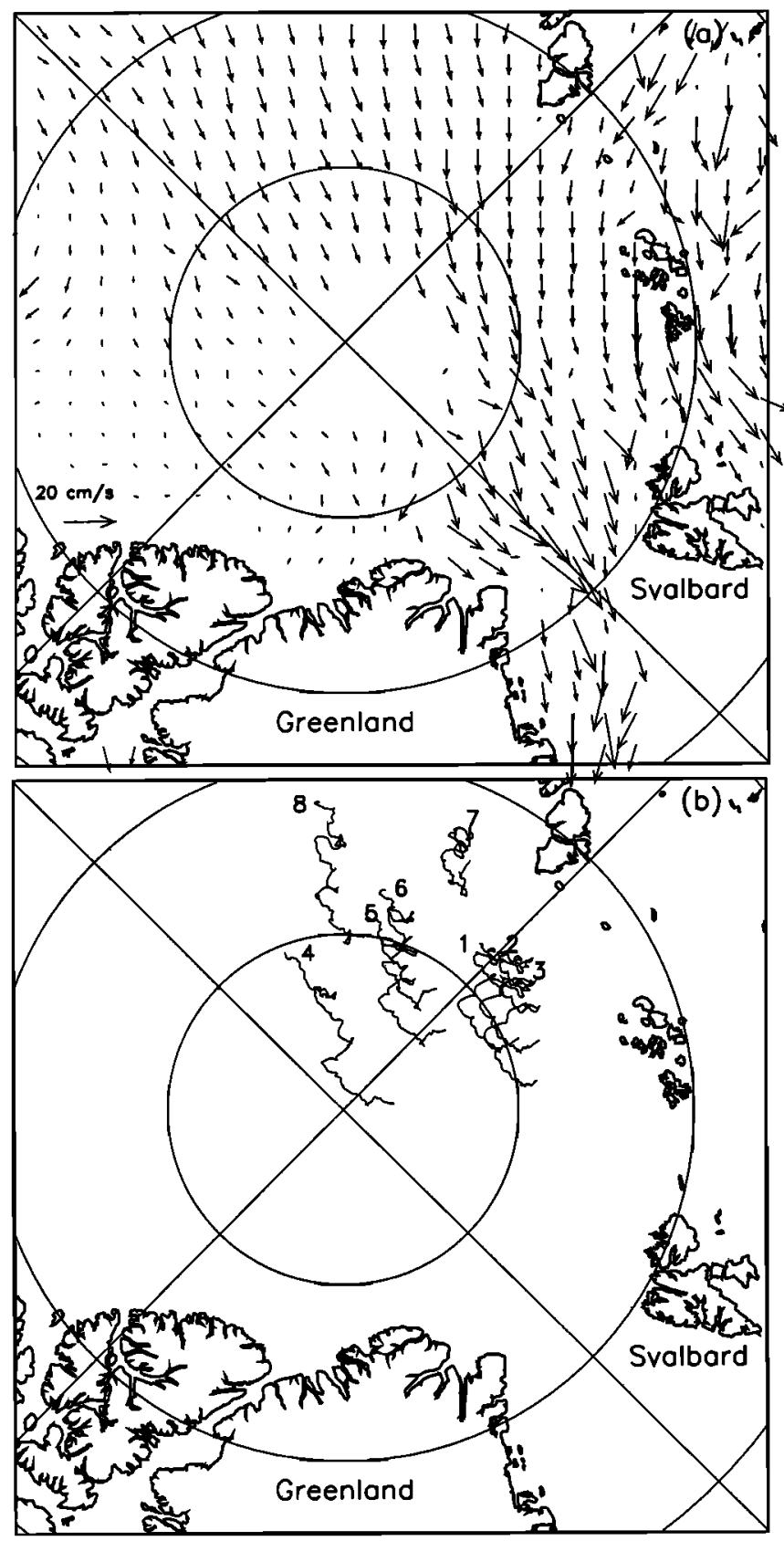

Figure 1. (a) Example from December 12, 1992 (day 73 relative to this data set), of velocity vectors derived from SSM/I brightness temperature using 2-D wavelet method described in text. (b) Selected buoys for the period October 1, 1992, to March 31, 1993, with buoy identification numbers represented as follows: 1,12819 ; 2,$12813 ; 3,1791 ; 4,2384 ; 5,2387 ; 6,9360 ; 7,1790$; and 8,9372 . Reference numbers are located near the start point of each buoy trajectory.

between positions such that $u=\Delta x / \Delta t, v=\Delta y / \Delta t$. Traditionally, this computation is made between consecutive buoy positions in time such that $u=x_{t}-x_{t-1} / \Delta t$ for $\Delta t=0.5$ days. Since this time step is significantly smaller than the one used to process the SSM/I data, we apply the SSM/I 4-day sliding window centered at a given time $t$ such that $u=x_{t+4}-x_{t-4} /(8 \Delta t)$. Gaps in the data not exceeding 3 days are linearly interpolated, while gaps greater than this are flagged as discontinuous and handled throughout the analysis as such. No prefiltering of either data set is done for this study as tidal and other resolvable high-frequency effects are found to be insignificant in deep basins.

\subsection{Case Study Selection}

By definition, deformation as a process describes both a change in shape and a change in size relative to some initial size. Examining the evolution of this change is an ideal way to compare two data sets. To do this, one needs to track features via some comoving frame of reference (Lagrangian) which in turn needs to be tracked within an Eulerian grid. The well-known tracking of Argos buoys within the context of a latitude, longitude frame is a clear illustration of such a combination. In this study, we take this process one step further by considering the sampling of satellite-derived vectors relative to the center of a buoy array.

Drinkwater [1998a,b], Kwok et al. [1998], and Liu and Zhao [1998] have made quantitative comparisons between buoy and satellite velocity in an Eulerian grid based on the configuration of satellite data fields. Complementary to these works, we consider the compatibility of buoy and satellite data sets relative to buoy tracks in order to examine the deformation. In this way, we access the relatively abundant source of satellite-gridded information through statistical sampling.

The Eurasian Basin is chosen as it has a relatively simple circulation (i.e., the Transpolar Drift), changes in the circulation pattern are primarily wind driven, and it is situated in deep water $(>1000 \mathrm{~m})$ at quite some distance from land. Such simplifications allow us to focus on the issues of scale and compatibility while minimizing such processes as boundary effects, tidal aliasing in the satellite signal, and variations in ocean circulation. Unfortunately, as seen from Figure 1a, this area is complicated by the lack of SSM/I data near the pole, and so buoy arrays that would cross this gap will not be considered. Given the above constraints, a total of eight buoys are found which span the full 6 month period as shown in Figure 1b. From these eight buoys, 11 arrays are constructed spanning in size from $100 \mathrm{~km}$ (using the three closest buoys) to $600 \mathrm{~km}$ (using all eight buoys) as listed in Table 1. While there are many more possible combinations of arrays which can be constructed, the 11 cases are judiciously chosen to represent the range of possible scales. As we prefer to examine deformation from a statistical rather than deterministic perspective, we include only one three-buoy array because of its relatively small size.

\subsection{Data Uncertainty}

Buoys are essentially point measurements in both space and time. Their primary source of error is ge- 
Table 1. Description of Buoy Arrays

\begin{tabular}{|c|c|c|c|c|c|c|}
\hline \multirow[t]{2}{*}{ Case } & \multirow{2}{*}{$\begin{array}{l}\text { Number } \\
\text { of Buoys }\end{array}$} & \multirow{2}{*}{$\begin{array}{c}\text { Buoys } \\
\text { Selected }\end{array}$} & \multirow{2}{*}{$\begin{array}{l}\text { Average } \\
\text { Size, km }\end{array}$} & \multicolumn{2}{|c|}{$\mathrm{S} / \mathrm{N}$} & \multirow[b]{2}{*}{$\mathrm{K}$} \\
\hline & & & & Velocity & Deformation & \\
\hline 1 & 3 & $1,2,3$ & 93. & - & - & 347 \\
\hline 2 & 4 & $4,5,6,8$ & 318. & 28.65 & 3.79 & 364 \\
\hline 3 & 4 & $1,5,6,7$ & 361. & 27.83 & 4.45 & 362 \\
\hline 4 & 4 & $1,2,6,7$ & 395. & 22.26 & 3.82 & 362 \\
\hline 5 & 4 & $5,6,7,8$ & 417. & 17.91 & 2.91 & 362 \\
\hline 6 & 4 & $1,3,4,5$ & 588. & 12.54 & 2.09 & 362 \\
\hline 7 & 5 & $1,2,3,4,5$ & 588. & 21.35 & 3.53 & 362 \\
\hline 8 & 5 & $1,3,4,5,6$ & 588. & 16.51 & 2.97 & 362 \\
\hline 9 & 6 & $1,4,5,6,7,8$ & 516. & 10.35 & 2.18 & 362 \\
\hline 10 & 7 & $1,3,4,5,6,7,8$ & 588. & 10.54 & 2.52 & 362 \\
\hline 11 & 8 & $1,2,3,4,5,6,7,8$ & 588. & 12.43 & 2.93 & 362 \\
\hline
\end{tabular}

$\mathrm{S} / \mathrm{N}$ is the signal-to-noise ratio as defined in the text. $\mathrm{K}$ is the number of times the strain rate is computed out of 364 twice daily measurements. The primary restriction is a minimum of three points.

olocation uncertainty $(300 \mathrm{~m})$ equivalent to $0.09 \mathrm{~cm}$ $\mathrm{s}^{-1}$ with a 4-day sliding window. SSM/I derived velocity vectors have essentially three sources of uncertainty. First, SSM/I vector data are produced for each day, but their motion is defined via a 4-day sliding window using features 2 days prior to and 2 days following a given day. The sliding window works as a filter to smooth out noise incurred by the SSM/I daily sampling and scanning techniques. As an upper limit, the temporal uncertainty for a given image is 1 day or $25 \%$. For a typical mean sea ice speed around $10 \mathrm{~cm} \mathrm{~s}^{-1}$, this equates to a maximum uncertainty of $2.5 \mathrm{~cm} \mathrm{~s}^{-1}(25 \%)$. The second source of uncertainty comes from the template matching, the size of which is one half the resolution of the data, that is, $6.25 \mathrm{~km}$ per 4 days or $1.8 \mathrm{~cm} \mathrm{~s}^{-1}$. A third uncertainty is the geolocation error of $8 \mathrm{~km}$ for $\mathrm{SSM} / \mathrm{I}$ [Kwok et al., 1998] which is equivalent to $2.3 \mathrm{~cm}$ $\mathrm{s}^{-1}$ with a 4-day sliding window.

As these are all uncorrelated sources of error, the overall error is expected to be $3.84 \mathrm{~cm} \mathrm{~s}^{-1}$, but this appears to be an upper limit as initial comparisons between SSM/I derived ice velocity and Argos buoys for the Arctic Ocean as reported by Liu et al. [1998] show better overall agreement $\left(2.52 \mathrm{~cm} \mathrm{~s}^{-1}\right.$ RMS difference (RMSD) for speed). With a longer sliding time window, the uncertainty is decreased, but the RMSD against buoys may increase because of feature tracking difficulties due to increased changes in ice dynamics and ice floe shape changes from ridging and melting processes. In comparison, the temporal and spatial uncertainties described above $\left(2.5,1.8\right.$, and $2.3 \mathrm{~cm} \mathrm{~s}^{-1}$, respectively) are smaller than the typical regional sea ice drift and compatible with the RMSD. The averaging of the three wavelet band-pass filtered scales may help in keeping the total error lower than overall expected error.

We make use of the buoy data to check the effectiveness of the 4-day sliding window. Using all eight buoys, average speed power density spectra (Figure 2) is computed as $\left(\operatorname{FFT}(u)^{2}+\operatorname{FFT}(v)^{2}\right) /(2 \Delta f)$ where FFT is the fast Fourier transform and $\Delta f$ is the frequency bin width [Geiger et al., 1998a]. This form of spectra represents the average kinetic energy per unit mass and bin width. Individual spectra (not shown) are similar in amplitude to these averaged results. Also included is the average surface geostrophic wind speed power density spectra using IABP surface pressure gradients from 55 points located between $80^{\circ}-88^{\circ} \mathrm{N}$ and $50^{\circ}-150^{\circ} \mathrm{E}$. The similarity between the surface geostrophic wind and buoy spectra support our assumption that sea ice mo-

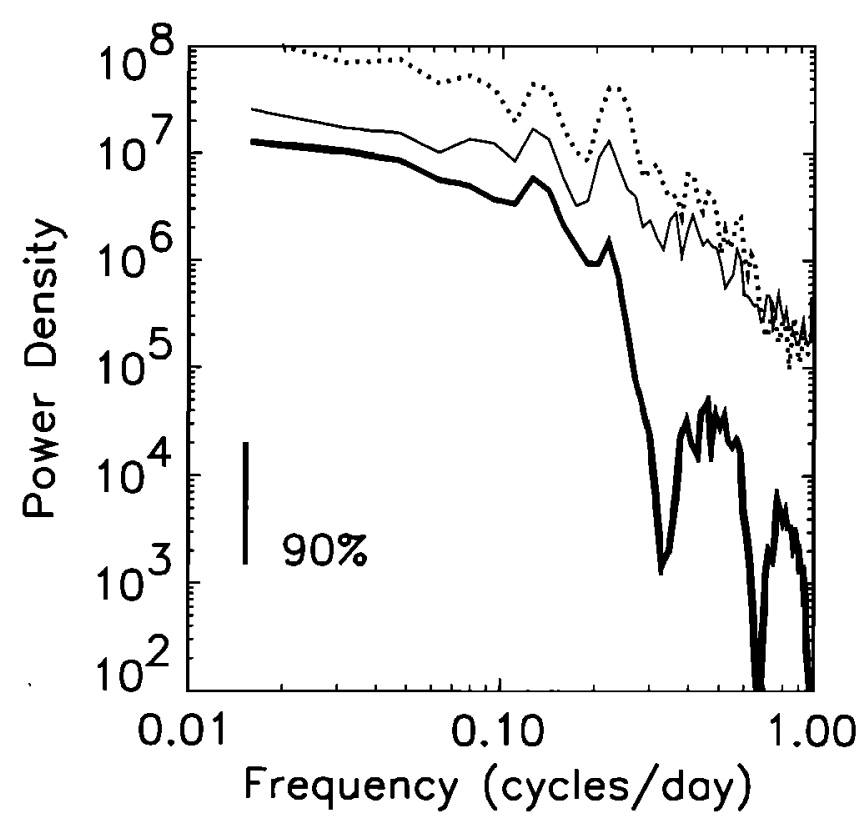

Figure 2. Ice and wind speed power density spectra using 64 frequency bins (bin widch $\Delta f=0.0077$ cycles $\left.\mathrm{d}^{-1}\right)$. The spectra $\left(\mathrm{cm}^{2} \mathrm{~s}^{-1}\right)$ is averaged from all eight buoys processed from consecutive positions using $\Delta t=0.5$ days (thin line), the 4-day sliding window (thick line), and nearby surface geostrophic wind (dotted). The $90 \%$ confidence $\chi^{2}$ is also indicated. Details described in text. 
tion in this region is dynamically wind dominated. Only at frequencies greater than 0.5 cycles $\mathrm{d}^{-1}$ ( $<2$ days) is this not the case, and the 4-day sliding window seems to adequately remove this.

In terms of filter performance, some loss of energy is found even at low frequencies $\left(<0.15\right.$ cycles $\left.d^{-1}\right)$, though still within the $90 \%$ confidence interval, with slightly greater loss from 0.15 and 0.25 cycles $\mathrm{d}^{-1}$. Significant drops in energy occur beyond $0.25{\text { cycles } \mathrm{d}^{-1}}^{-1}$ (4 days). As an artifact of the sliding window, there are also two significant peaks introduced near 0.45 and 0.80 cycles $\mathrm{d}^{-1}$ but in both cases these are nearly 2 orders of magnitude below the original signal. While not a perfect filter, the 4-day sliding window seems to remove much of the higher frequency motion in the 12-hour buoy data. Hence, using the assumptions and preprocessing described in this section, a comparison between the SSM/I and buoy data sets is successfully accomplished at the expense of losing high frequency variability in the buoy signal $>0.25$ cycles $\mathrm{d}^{-1}$ ( $<4$ days).

\section{Methodology}

We apply a method used in buoy strain rate analysis as described by Geiger et al. [1998a] where local velocity and deformation components are computed under the assumption of 2-D large-scale motion relative to a collection of comoving particles at any given time by the Taylor expansion about the point $x_{0}, y_{0}$ of

$$
v_{n i}=v_{0 i}+\frac{\partial v_{i}}{\partial x_{j}} \Delta x_{n j}+E_{v_{i}}
$$

In this form repeated indices sum; $i, j=1,2$ represent the $x, y$ coordinates; $n=1, \cdots, N$ particles; $v_{n i}$ are the observed velocities at positions $x_{n}, y_{n}$ with
$x_{n}=x_{0}+\Delta x_{n}$ and $y_{n}=y_{0}+\Delta y_{n} ; v_{0 i}=\left(u_{0}, v_{0}\right)$ are local velocity components; and $\partial v_{i} / \partial x_{j}$ are the four deformation components. The solution to (1) in the absence of $E_{v_{\mathrm{v}}}$ is deterministic and exact for $N=$ three particles(six equations and six unknowns: $v_{0 i}, \partial v_{i} / \partial x_{j}$ ). For more than three particles the solution is found statistically via multiple linear regression [Geiger et al., 1998a] given the known positions $\Delta x_{n j}=\left(x_{n}, y_{n}\right)$ and velocities $v_{n i}=\left(u_{n}, v_{n}\right)$ from a number of particles. Once the local velocity and deformation components are determined, invariant quantities of speed $(|\vec{V}|)$, divergence $(D)$, and maximum shear $(S)$ follow from

$$
\begin{aligned}
|\vec{V}| & =\sqrt{u_{0}^{2}+v_{0}^{2}} \\
D & =\frac{\partial u}{\partial x}+\frac{\partial v}{\partial y} \\
S & =\sqrt{\left(\frac{\partial u}{\partial x}-\frac{\partial v}{\partial y}\right)^{2}+\left(\frac{\partial u}{\partial y}+\frac{\partial v}{\partial x}\right)^{2}} .
\end{aligned}
$$

Following Geiger et al. [1998a] and Hines and Montgomery [1990], we check the quality of the statistical solution via a signal-to-noise ratio of the solved quantities $v_{0 i}, \partial v_{i} / \partial x_{j}$ where the signal is the estimated value of the unknowns as found from the regression solution to (1) and the noise is estimated from

$$
\begin{aligned}
C I_{i m} & =t_{\alpha / 2, \nu} \sqrt{\sigma_{i}^{2} C_{m m}} ; \\
\sigma_{i}^{2} & =\frac{1}{N} \sum_{n}\left(v_{n i}-\hat{v}_{n i}\right)^{2} ;
\end{aligned}
$$

\begin{tabular}{|c|c|c|c|c|c|c|c|c|c|c|}
\hline \multirow[t]{2}{*}{ Case } & \multirow{2}{*}{$\begin{array}{l}\text { Optimal } \\
\text { Radius }\end{array}$} & \multirow{2}{*}{$\begin{array}{c}\text { Array } \\
\text { Size }\end{array}$} & \multicolumn{3}{|c|}{ Velocity } & \multicolumn{3}{|c|}{ Deformation } & \multirow[b]{2}{*}{$\mathbf{P}$} & \multirow[b]{2}{*}{$\mathrm{K}$} \\
\hline & & & $S / N$ & RMSD & $\bar{\rho}$ & $\overline{S / N}$ & RMSD & $\rho$ & & \\
\hline 1 & 550. & 995. & 11.28 & 2.81 & 0.89 & 3.60 & 15.23 & 0.51 & 65 & $\overline{171}$ \\
\hline 2 & 650. & 1066. & 13.92 & 0 & 0.90 & 4.40 & 8.03 & 0.72 & 90 & 180 \\
\hline 3 & 550. & 974. & 12.75 & 2.52 & 0.89 & 3.89 & 8.43 & 0.74 & 69 & 177 \\
\hline 4 & 550 & 986. & 12.21 & 2.51 & 0.90 & 3.81 & 8. & 0.76 & 68 & 177 \\
\hline 5 & 600. & 1031. & 14.18 & 2.42 & 0.90 & 4.53 & 7.8 & 0.71 & 84 & 177 \\
\hline 6 & 60 & 1069. & 12.49 & 2.45 & 0.89 & 3.96 & 10. & 0.74 & 74 & 178 \\
\hline 7 & 550 & 986. & 12.16 & 2.50 & 0.89 & 3.64 & 10.15 & 0.74 & 64 & 177 \\
\hline 8 & 5 & you. & 12.38 & 2.41 & 0.89 & 3.57 & 8.05 & 0.77 & 62 & 176 \\
\hline 9 & 600. & 1026. & 13.67 & 2.34 & 0.89 & 4.25 & 6. & 0.81 & 80 & 177 \\
\hline 10 & 650 & 1160. & 13.43 & 2.28 & 0.90 & 4.51 & 5.8 & 0.83 & 92 & 177 \\
\hline 11 & 650 & 1172. & 13.15 & 2.31 & 0.90 & 4.49 & 5.97 & 0.83 & 91 & 177 \\
\hline
\end{tabular}

where $C I_{i m}$ is a confidence interval matrix arranged as coordinate $(i)$ by expansion variable $(m)$ for $m=3$ ( $v_{0 i}, \partial v_{i} / \partial x_{j}$; for $i, j=1,2$ coordinates), $t_{\alpha / 2, \nu}$ is the

Table 2. Optimal SSM/I Results Relative to Buoy Arrays

The optimal radius (kilometer) is the search radius found for the minimum RMSD value. The array size (kilometer) is defined here as the average maximum distance between all possible points of an array. For the velocity and deformation components, $\mathrm{S} / \mathrm{N}$ is the signal-to-noise ratio as defined in the text; RMSD is the RMS vector difference as per equation (10) between SSM/I and buoy arrays at the buoy array centroid in units of $\mathrm{cm}$ $\mathrm{s}^{-1}$ for velocity and $10^{-8} \mathrm{~s}^{-1}$ for deformation; and $\rho$ is the correlation coefficent found at the RMSD minimum. $P$ is the average number of SSM/I vectors found within the chosen search radius during the $K$ times the strain rates are compared out of 180 days. 
student's $t$ value at a probability $1-\alpha$, and $\nu$ is the degrees of freedom equal to $2 N-6$. The $C_{m m}$ term inside the square root is the diagonal of the covariant matrix, and $\hat{v}_{n i}$ is the velocity of the known particles estimated by the regression solution.

For this study, the signal-to-noise $(\mathrm{S} / \mathrm{N})$ as shown in Tables 1 and 2 is based on the $90 \%$ confidence interval $(1-\alpha=0.9)$. The $\mathrm{S} / \mathrm{N}$ is computed at every time step for each expansion variable (e.g., $u_{0} / C I_{11}$ ), added as a vector (i.e.,

$$
\text { and } \begin{aligned}
& \sqrt{\left(u_{0} / C I_{11}\right)^{2}+\left(v_{0} / C I_{21}\right)^{2}} \\
& \sqrt{\left.\sum_{i, j=1}^{2}\left(\left(\partial v_{i} / \partial x_{j}\right) / C I_{i, j+1}\right)^{2}\right)}
\end{aligned}
$$

and then averaged over the length of each time series.

\subsection{Buoy Strain Rate}

Table 1 lists the arrays and corresponding buoys. The location $x_{0}, y_{0}$ is defined by the centroid of each array. For the first case, three buoys are used, and the solution is deterministic such that the noise level and signal-tonoise are theoretically zero and infinite, respectively. For the cases with four or five buoys the signal-to-noise levels were found to be artificially high for all six components (i.e., the confidence levels were artificially low). Using 6 buoys, the signal-to-noise level reaches a minimum of 10.35 and 2.18 for the average velocity and deformation components, respectively, with an increase to 12.43 and 2.93 , respectively, when eight buoys are used.

Similar variations in the signal-to-noise are noticed in the SSM/I cases constructed later with minimum signal-to-noise found for 8-10 SSM/I points (search radius $200-250 \mathrm{~km}$ ). There are two possible sources for these variations, either the limited number of buoys or the size of the array (influence of a smaller $\Delta x_{n j}$ ). As seen in Table 1 for the buoy cases, array sizes using four to five buoys are, in some cases, the same size as the six to eight buoy arrays (cases 5-7 versus 8-10, respectively) and hence the limited number of buoys seems to be the prevalent cause of the unusually high signalto-noise values in cases $2-7$. The end result is that six or more buoys are needed before the signal-to-noise ratio can be used as a justifiable measure of the quality of the solution. These results corroborate with earlier findings by Thorndike [1986] who also found 6 buoys to be a practical statistical minimum.

\subsection{SSM/I Strain Rate}

A number of methods were attempted in an effort to compare the SSM/I and buoy strain rates. During these attempts it was found that whenever interpolation was used to transform data from one system to another, there was a loss in spatial variability due to weighted averaging in the interpolation process. Thus interpolation invokes a smoothing which is detrimental in the computation of deformation. In hindsight, this is to be anticipated as it is the variability of the velocity field which defines the deformation. To bypass this effect we found it essential to sample rather than average the SSM/I velocity field in order to estimate the deformation with any degree of certainty. The following was found to be the most effective method given the resources available to us.

First, at a given time, we choose some known position $\left(x_{0}, y_{0}\right)$ such as the center of a moving buoy array. Next, we identify from a gridded field of SSM/I data nonzero velocity points located within a distance $R$ of $x_{0}, y_{0}$. Then, using the multiple linear regression method described earlier, we compute the local field deformation at $x_{0}, y_{0}$ using known positions $\Delta x_{n j}$ and velocities $v_{n i}$ for $i=1, \cdots, N \mathrm{SSM} / \mathrm{I}$ grid points. In this way we treat the gridded data like an array of particles which we sample at each time relative to a chosen scale size $R$ and location $x_{0}, y_{0}$. Unlike a buoy array, the number of SSM/I gridded samples will vary in time and will also not continuously reference the same particles. This being the case, the classic approach to deformation using a triangular array must be replaced by a statistical method of $N$ points for $N \gg 3$. We direct our methodology in this way because it provides a means for combining gridded satellite data with multiple or single buoy tracks to produce localized material deformation information about large-scale sea ice processes.

The only difficulty with this method is the determination of $R$ which must be judiciously chosen, preferably relative to some statistically and geophysically based scale size. Hence, relative to the center of each array listed in Table 1, we compute SSM/I strain rates using a range of 20 possible spatial scales from 100 to 1050 $\mathrm{km}$ at $50 \mathrm{~km}$ intervals giving $220 \mathrm{SSM} / \mathrm{I}$ strain rate solutions. Results from these solutions are compared against the 11 buoy arrays whose scales vary from 100 to $600 \mathrm{~km}$.

\subsection{Determination of Optimal Scale}

The RMSD between any two scalar data sets $\left(S^{(1)}\right.$, $\left.S^{(2)}\right)$ is given by

$$
\mathrm{RMSD}=\sqrt{\frac{1}{K} \sum_{k}\left(S_{k}^{(1)}-S_{k}^{(2)}\right)^{2}}
$$

where the superscripts in parentheses distinguish the two data sets whose time series are of length $k=$ $1, \cdots, K$. Applying the same principle to vector and deformation components based on vector difference we get

$$
\mathrm{RMSD}=\sqrt{\frac{1}{K} \sum_{k} \sum_{n}\left(S_{n k}^{(1)}-S_{n k}^{(2)}\right)^{2}}
$$

for $n=1, \cdots, N$ components (i.e., $N=2$ for velocity vector difference, $N=4$ for deformation vector differ- 


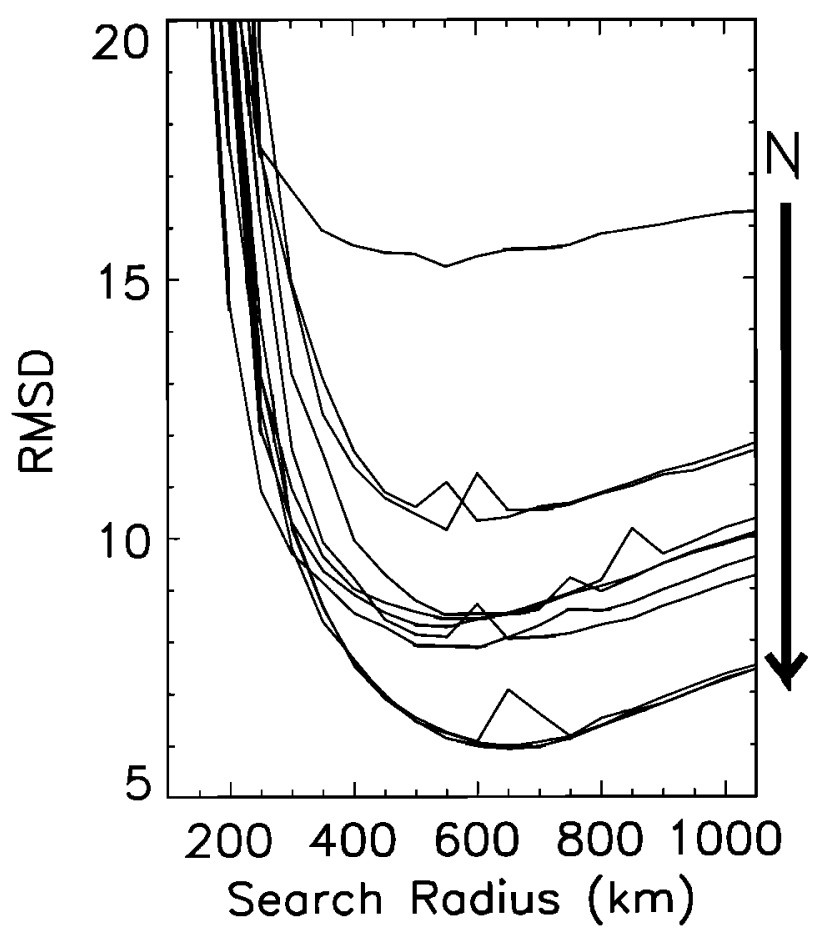

Figure 3. RMSD between SSM/I and buoy deformation $\left(10^{-8} \mathrm{~s}^{-1}\right)$ using (8). Each line represents RMSD results between each buoy array and SSM/I data sampled over a range of search radii. The $\mathrm{N}$ and corresponding arrow to the right of the plot are used to illustrate how an increasing number of buoys per array reduce the overall $\mathrm{RMSD}$.

ence). As will be shown in section 5.1, the RMSD for invariant quantities are larger than the RMSD vector computations using (10) owing to propagated uncertainties. It is for this reason that we examine the vector RMSD from the regression estimates directly.

The smaller the RMSD between two data sets, the better they compare. Since it is the scale of the spatial variability we are most interested in, the RMSD of the four deformation components using (10) is the most critical to minimize as it is these components which make up the pieces that determine the strain rate invariants as expressed in (3) and (4). A plot of deformation RMSD for the 11 cases is shown in Figure 3. Note first the rapid reduction in RMSD from the smallest search radius to about $400 \mathrm{~km}$. Second, the RMSD minimum is not a sharp feature but a broad minimum centered around $600 \mathrm{~km}$. Third, as the number of buoys and size of the buoy arrays increase, the RMSD decreases with a slight shift in RMSD minimum from about 550 to about $650 \mathrm{~km}$. Some spiking is seen in all but the smallest (upper line) and two largest (lower lines) cases which may be a result of either a transition in scale or too few buoys in the statistical solution.

A detailed listing of the best result for each case is shown in Table 2 . In addition to minimizing the RMSD, a scale based on maximum correlation coeffi- cient was computed. Results from that analysis (not shown here) are similar to those found for the RMSD (varying in only three cases by $50-100 \mathrm{~km}$ from the minimum RMSD scale). At the minimum RMSD, the RMSD via (10) from all 11 arrays is $2.44 \pm 0.05 \mathrm{~cm} \mathrm{~s}^{-1}$ for velocity and $8.6 \pm 0.8 \times 10^{-8} \mathrm{~s}^{-1}$ for deformation with corresponding correlation coefficients of $0.895 \pm 0.001$ and $0.741 \pm 0.027$, respectively.

Table 2 also shows an SSM/I array size of the order of $1000 \mathrm{~km}$. Recalling that the size of the buoy arrays range from 100 to $600 \mathrm{~km}$, it is noteworthy to find such a large SSM/I array size. This size scales more with atmospheric storms and is hypothesized to be geophysically based as noted in earlier works [e.g., Thorndike and Colony, 1980].

\section{Comparison Results}

An average search radius of $600 \mathrm{~km}$ corresponding to an SSM/I sample size of $1000 \mathrm{~km}$ is found to yield the best result when trying to find a compatible scale to compare buoy and SSM/I strain rate arrays in the Eurasian Basin. This length scale is found in accordance with minimizing RMSD for deformation, maximizing the correlation of the deformation components, and in agreement with the length scale of the dominant forcing. At this scale the average RMSD (2.48 土 $0.05 \mathrm{~cm} \mathrm{~s}^{-1}$ for velocity vector and $8.8 \pm 0.9 \times 10^{-8}$ $\mathrm{s}^{-1}$ using all four deformation components via (10) is only slightly larger than the minimum RMSD using a variable scale. The corresponding correlations $(0.896$ \pm 0.002 and $0.729 \pm 0.030$, respectively) are also only slightly less than those using a variable optimal scale. An example result is shown in Figure 4.

To better understand how this optimization works, we use the following illustration. At a search radius of $600 \mathrm{~km}$, the time series for invariants using SSM/I vectors will look like those in Figure 4. Decreasing the search radius of the SSM/I will produce time series of these invariants with much larger amplitudes, taking on the appearance of a noisy signal. Conversely, as the $\mathrm{SSM} / \mathrm{I}$ search radius is increased beyond $600 \mathrm{~km}$, amplitudes of the SSM/I will approach a flat line along zero. Hence, near $600 \mathrm{~km}$, a balance between noise in the data set and smoothing associated with the inclusion of more points reaches an optimum where the two signals (SSM/I and buoy arrays) are closest to each other.

As seen in Table 2, the signal-to-noise ratios for the $\mathrm{SSM} / \mathrm{I}$ at the optimal scales, are slightly larger than those of the largest buoy array (12.43 and 2.93 for buoy velocity and deformation, respectively) despite the fact that there are, on average, 10 times more data points per calculation for the SSM/I (Table 2). Thus there is comparable noise in both data sets. The larger signalto-noise ratio in velocity over deformation (factor of 3) furthermore exemplifies how the velocity uncertainties from both data sets are amplified as they propagate 

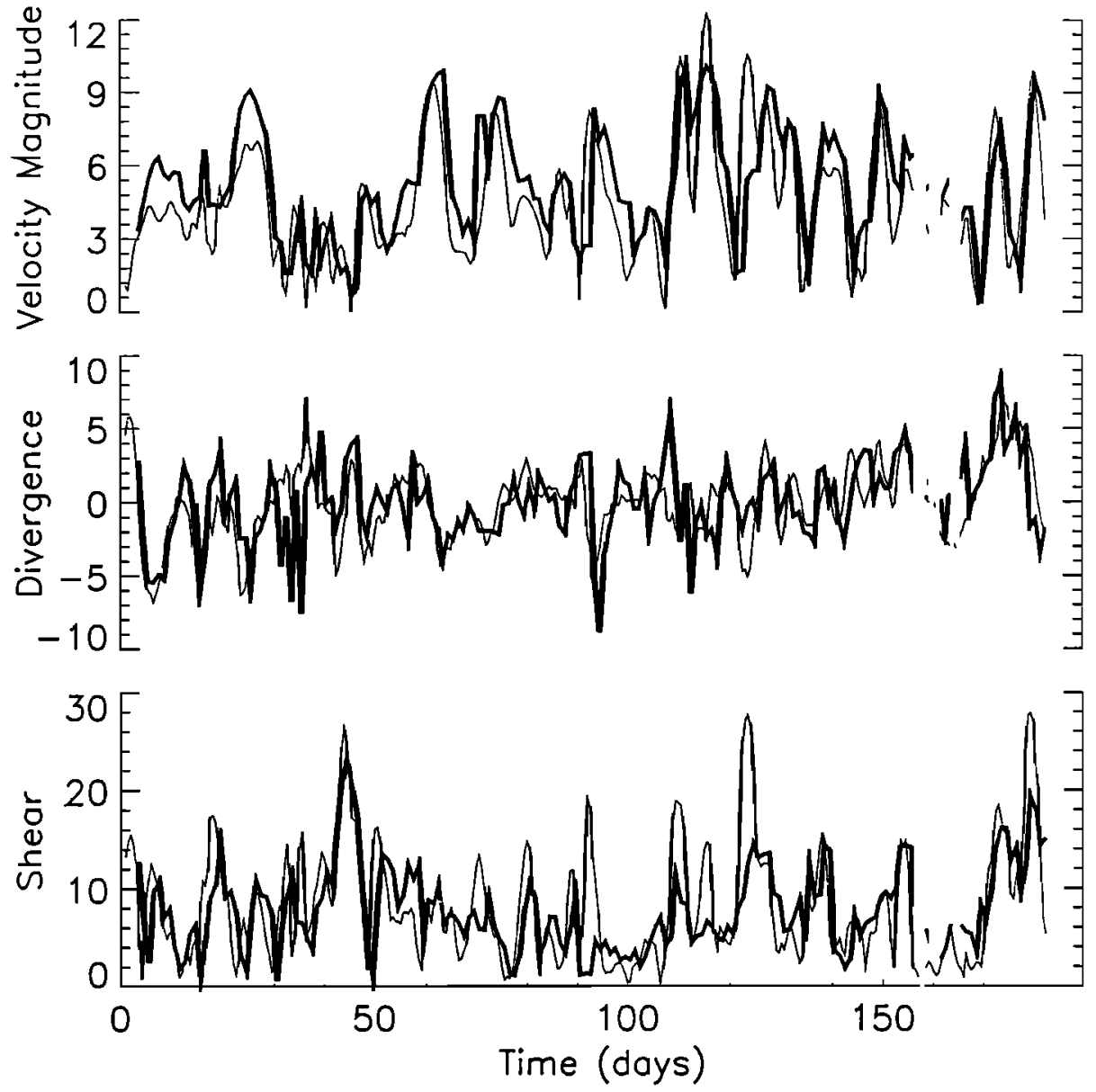

Figure 4. Results from case 11 using all eight buoys (thin line) and a $600 \mathrm{~km}$ search radius for the SSM/I (thick line) for velocity magnitude $\left(\mathrm{cm} \mathrm{s}^{-1}\right)$, divergence, and shear $\left(10^{-8} \mathrm{~s}^{-1}\right)$.

through the computation of differential terms and further still to the invariant calculations. Corroborating with this result, correlations of 0.9 between buoys and $\mathrm{SSM} / \mathrm{I}$ average velocity components (Table 2 ) were only able to attain a correlation of 0.83 for the best case (Table 2, case 11) for deformation and less than that for the invariants (e.g., divergence 0.56, see Figure 5). This clearly demonstrates the need for the most accurate satellite-derived motion data possible if one is to consider these velocity fields for mass balance type applications.

Focusing on the outcome near the optimal scale, we wish to understand how well SSM/I and buoy results compare and moreover determine how such results could be improved further. Using the example shown in Figure 4, we look at the scatter in Figure 5. A perfect fit would match the diagonal while distance from the diagonal is measured by the size of the RMSD between the two data sets. Liu and Zhao [1998] found an RMSD for speed of $2.52 \mathrm{~cm} \mathrm{~s}^{-1}$ in the Arctic (not including the area east of Svalbard and Franz Josef Land). RMSD results here suggest an RMSD speed of $1.83 \mathrm{~cm} \mathrm{~s}^{-1}$. Unlike earlier works by Liu which included the entire
Arctic Basin, this local study shows a bias with SSM/I slightly overestimating speed relative to the buoy arrays. Probability distribution functions using all 11 cases (Figure 6a) provide some explanation of this bias. Low speeds are under predicted in the SSM/I relative to the buoys, while higher speeds are over predicted with the tail of the buoy distribution reaching higher speeds.

For direction no bias is found as seen in both Figures $5 \mathrm{~b}$ and $6 \mathrm{~b}$ with RMSD, and is close to that found by Liu and Zhao [1998]. An average RMSD based on all 11 cases is only slightly higher than results reported for this case, at $1.89 \pm 0.03 \mathrm{~cm} \mathrm{~s}^{-1}$ and $33.01^{\circ} \pm 1.42^{\circ}$ for speed and direction, respectively, with the same bias in speed as seen for this example.

For the invariants of divergence and invariant shear, we find encouraging results (RMSD of $2.47 \times 10^{-8} \mathrm{~s}^{-1}$ and $4.56 \times 10^{-8} \mathrm{~s}^{-1}$, respectively). Figure 5 shows only a small bias in the shear at larger values. The RMSD for divergence is half the size of the RMSD for shear, but the divergence magnitude is also considerably smaller (factor of 3) than shear and so the RMSD roughly scales relative to the magnitude. Figure 6 shows SSM/I shear values over estimated in the midrange $\left(4-14 \times 10^{-8} \mathrm{~s}^{-1}\right)$ 

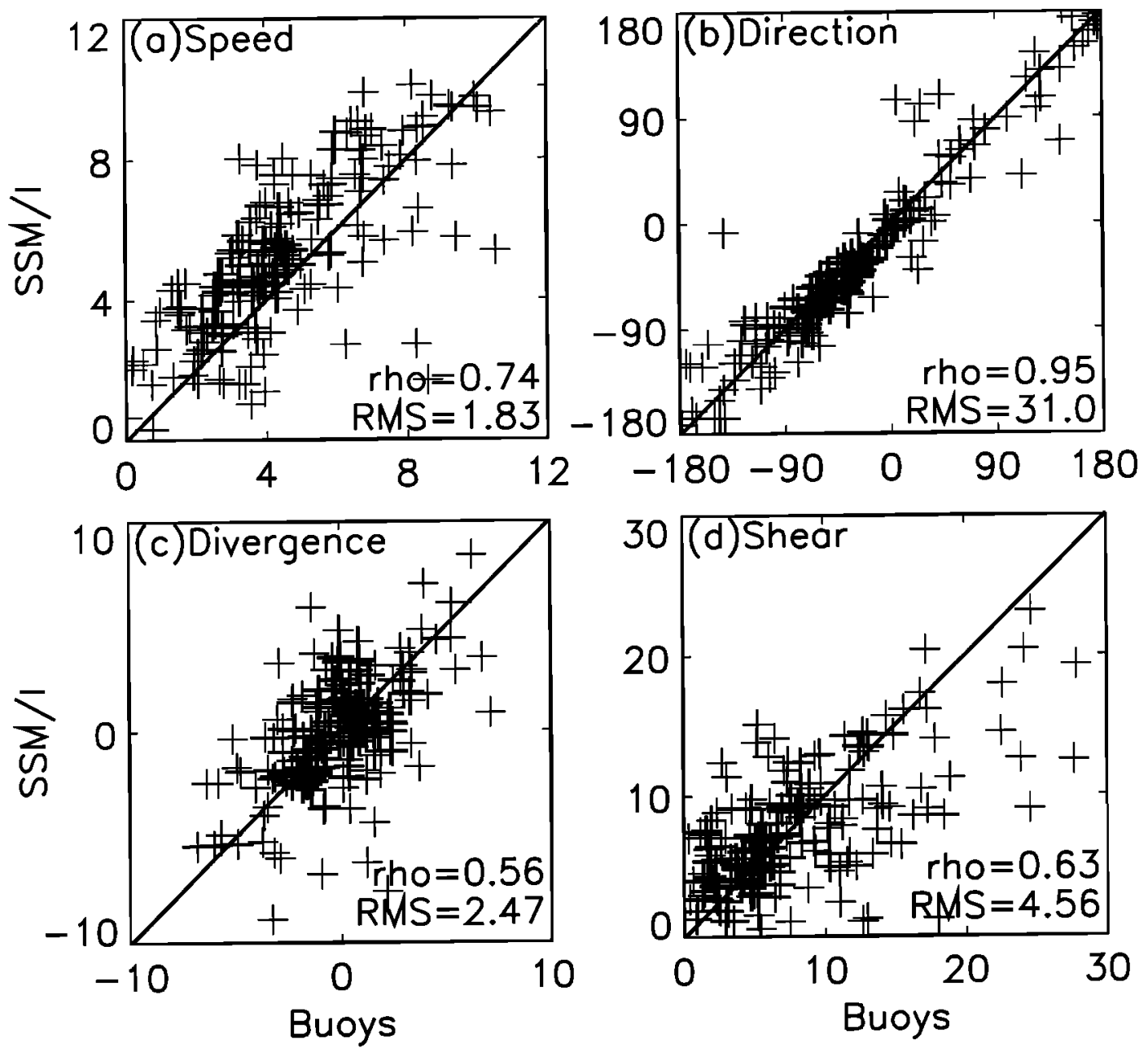

Figure 5. Scatter plots from case 11 based on all eight buoys and SSM/I using a $600 \mathrm{~km}$ search radius. Velocity (a) speed and (b) direction are in units of $\mathrm{cm} \mathrm{s}^{-1}$ and degrees, respectively; (c) divergence and (d) invariant shear are in units of $10^{-8} \mathrm{~s}^{-1}$. The RMSD and correlation (rho) for SSM/I-buoy are indicated in the lower right-hand corner of each property.

and under estimated at high magnitudes compared with the buoys. For divergence (Figures $5 c$ and $6 c$ ) the large scatter relative to its magnitude is the major discrepancy.

The speed and invariants of divergence and shear (Figure 7) show the primary differences in frequency space, the speed being computed as for Figure 2 with an analogous method applied to each of the components in (3) and (4) as described by Geiger et al. [1998a]. For all but the divergence, the low-frequency peaks, while not significant at the $90 \%$ confidence interval, are similar in amplitude at corresponding frequencies. For divergence the midrange frequencies between 0.1 and 0.2 cycles $d^{-1}$ (10 and 5 days, respectively) correspond well. The lowest frequencies $\left(<0.03\right.$ cycles $\left.\mathrm{d}^{-1}\right)$ also match, but between these $\left(0.03\right.$ to 0.1 cycles $\left.\mathrm{d}^{-1}\right)$ they correspond less well in amplitude. The amplitude of the divergence is about an order of magnitude less than for the shear invariant, and so we suspect noise in the form of tem- poral and spatial uncertainties from both the SSM/I and buoy arrays will have their greatest impact on the divergence. The higher frequencies $\left(>0.15\right.$ cycles $\left.\mathrm{d}^{-1}\right)$ confirm our suspicions. Remnants of the artificial peak at 0.45 cycles $\mathrm{d}^{-1}$ seen in Figure 2 are also shown here. While these high frequency buoy peaks match SSM/I results, there is a drop in power at 0.25 cycles $\mathrm{d}^{-1}(4$ days) in the buoys and a peak near 0.2 cycles $d^{-1}$ in the SSM/I which do not match.

\section{Discussion and Conclusions}

A useful method for comparing drift and deformation between buoys and SSM/I is demonstrated for the Eurasian Basin with velocity results agreeing with earlier studies by Liu and Zhao [1998]. In terms of scale compatibility, the velocity and deformation results are based on an optimal search radius of $600 \mathrm{~km}$ corresponding to a length scale of $1000 \mathrm{~km}$ and temporal 

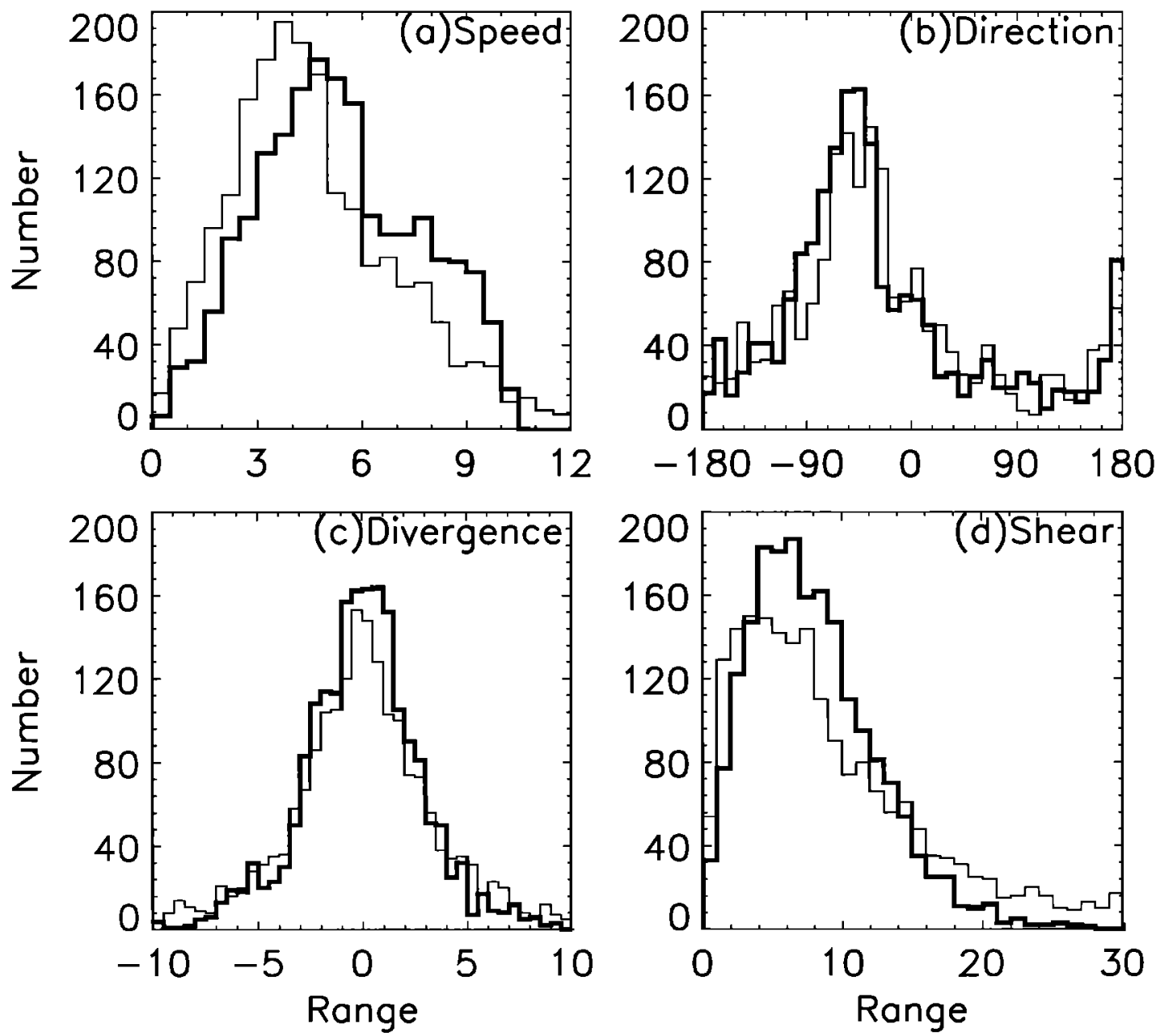

Figure 6. Probability distribution functions using all 11 case studies of the buoy arrays (thin line) and SSM/I grid using a $600 \mathrm{~km}$ search radius (thick line). The ranges for each property are given in units of (a) $\mathrm{cm} \mathrm{s}^{-1}$, (b) degrees, and (c) and (d) $10^{-8} \mathrm{~s}^{-1}$.

scales greater than 4 days. The sizes of this length scale and of atmospheric storms are too consistent to be coincidental.

$\mathrm{SSM} / \mathrm{I}$ and buoy velocity and deformation at the chosen scale compare well with correlations of $0.896 \pm 0.002$ and $0.729 \pm 0.030$, respectively. The relatively simple circulation of the Eurasian Basin has provided an excellent testing ground for this initial study, but length scales and results found here should not a posteriori be extended to other regions of the Arctic or Antarctic without further study. Additionally, because of the cascade of uncertainty from drift to differential drift and then onto deformation invariants, correlations between buoy and SSM/I in velocity which average 0.9 in nearly all cases could at best achieve a correlation of 0.56 for divergence (Figure 5c). Therefore care should be taken in applying such divergence results to mass balance estimates as the uncertainties exemplified by the low signalto-noise ratios (Table 2) for deformation are still quite high.

\subsection{Noise Sources}

In an effort to quantify some of the noise from both the buoys and SSM/I, we consider the following scaling arguments. For buoys, a geolocation error $(300 \mathrm{~m})$ equates to $U=0.09 \mathrm{~cm} \mathrm{~s}^{-1}$ using a 4-day sliding window. Using the length scale $L=1000 \mathrm{~km}$ found, deformation components should have corresponding uncertainties of $U / L \approx 0.09 \times 10^{-8} \mathrm{~s}^{-1}$. Propagating these errors [Beers, 1957], the uncertainty in divergence and invariant shear should be $0.13 \times 10^{-8} \mathrm{~s}^{-1}$ and $0.18 \times 10^{-8}$ $\mathrm{s}^{-1}$, respectively. Compared with values seen in Figures 4 and 5 , these errors are an order of magnitude below the typical signals of $5.0 \times 10^{-8}$ and $15.0 \times 10^{-8} \mathrm{~s}^{-1}$, respectively. Buoy array signal-to-noise ratios (roughly 10 and 3 for velocity and deformation signal-to-noise, respectively) equate to noise levels of 10 and $30 \%$, respectively. Compared with the geolocation uncertainty, these values are much larger meaning much of the noise remains unexplained. 

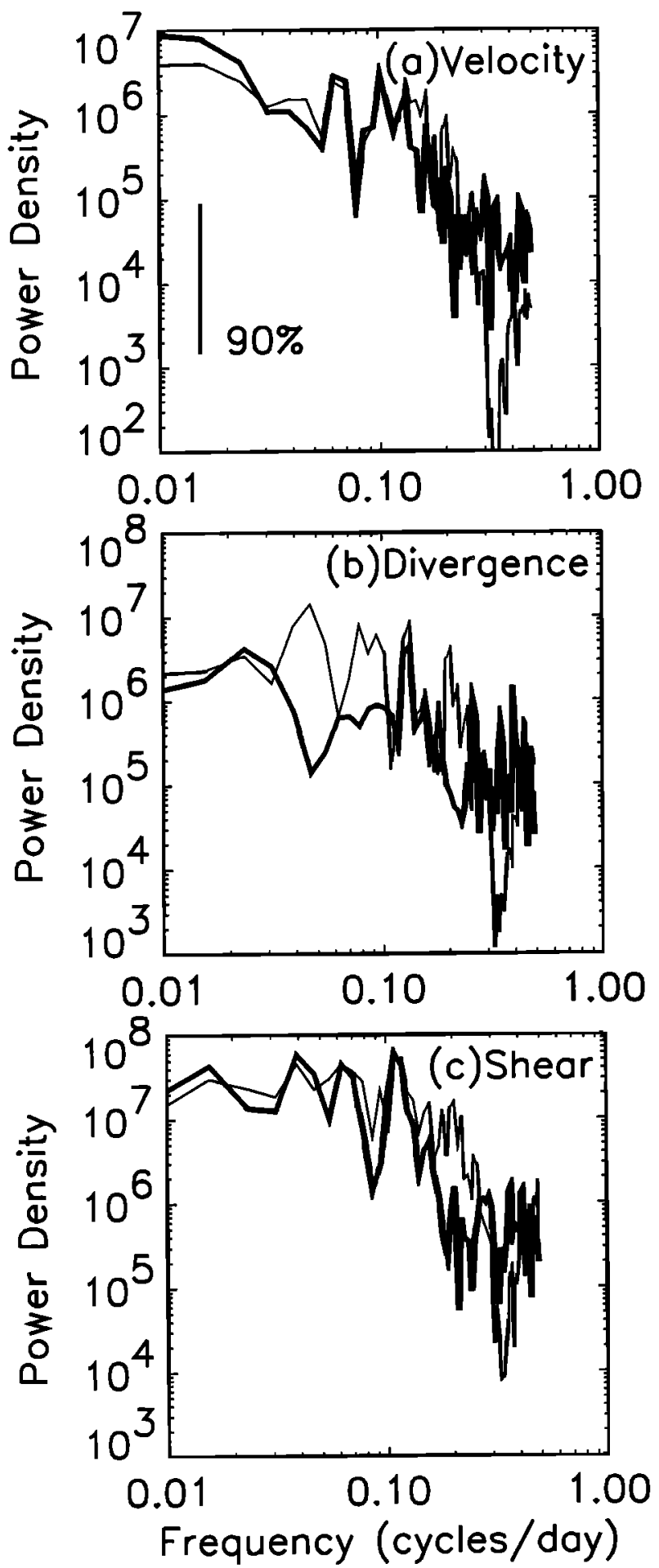

Figure 7. Average power density spectra from all 11 buoy cases (thin line) and SSM/I with $600 \mathrm{~km}$ search radius (thick line). Spectra are in units of squared amplitude per frequency bin width $(\Delta f=0.0077$ cycles $\left.\mathrm{d}^{-1}\right)$ for (a) speed $\left(\mathrm{cm}^{2} \mathrm{~s}^{-1}\right)$, (b) divergence $\left(10^{-16}\right.$ $\left.\mathrm{s}^{-1}\right)$, and (c) invariant (maximum) shear $\left(10^{-16} \mathrm{~s}^{-1}\right)$. Details provided in text.

Another source of uncertainty for the buoys is their small number. This is much more difficult to quantify but in essence represents the ability of an instrument to adequately cover a chosen spatial scale. Recalling the signal-to-noise results from section 4 for the six buoy array (10.35 and 2.18 for velocity and deformation, respectively) and eight buoy array (12.43 and 2.93 , respectively), noise levels decrease roughly 20 and $30 \%$, respectively, with an increase of just two buoys. Therefore the issue of buoy number appears to be greater as a noise source than the geolocation error at these temporal and spatial scales.

For SSM/I, uncertainty in velocity $\left(U=2.5 \mathrm{~cm} \mathrm{~s}^{-1}\right)$ propagates to $2.5 \times 10^{-8} \mathrm{~s}^{-1}$ for each deformation component and further to $3.6 \times 10^{-8} \mathrm{~s}^{-1}$ for divergence and $5.0 \times 10^{-8} \mathrm{~s}^{-1}$ for invariant shear. The RMSD found for divergence and shear shown in Figure 5 (2.47 and $4.56 \times 10^{-8} \mathrm{~s}^{-1}$, respectively) are of similar size.

In summarizing these scaling arguments, the noise sources identified in both the buoy and SSM/I arrays, while quite different in origin, appear to be of similar size for the spatial scales and timescales chosen. These uncertainties are concentrated at each time step or at least within the limits of the 4-day sliding window and as such represent high-frequency noise as supported by Figure 7.

\subsection{Utilizability for Future Studies}

Averaging velocity fields naturally corrupts the spatial variability of the field and therefore degrades deformation estimates. It is therefore of paramount importance that a distinction be made between noise/uncertain1 and natural variability. Given the importance of deformation to open water processes, methods of averaging satellite results to obtain mean motion only address part of the problem. The smoothed monthly mean velocity fields often sought by climate modelers yield precarious consequences when further used for computing open water fraction because of loss of important large-scale variability. Using these averages to further compute deformation or using these directly to consider mass balance products can yield misleading results.

Averaging (an even interpolation) of velocity fields should be kept to a minimum and only to scales significantly below the scale of field variability being investigated. Local deformation fields should be computed via sampling methods similar to the one described here. Then averages or probability distributions of strain rate properties (divergence, invariant shear, or shear direction) can be made to obtain the needed weekly or monthly inputs for modeling. The Gaussian shape of the divergence shown in Figure 6 and subcomponents for invariant shear of elongation and local shear (not shown) are similar in shape to results found in the western Weddell Sea during 1992 [Geiger et al., 1998b] for each of the deformation components, suggesting a Gaussian distribution of these components as a useful model parameterization.

Liu and Zhao [1998] have already derived velocity vectors from the NASA Scatterometer (NSCAT) data with results from NSCAT and SSM/I found to be compatible. The NSCAT coverage is complementary to the 
SSM/I when there are heavy clouds or surface effects. This outcome allows for three sea ice drift daily results (i.e., NSCAT, SSM/I, and buoys) merged by data fusion techniques to form a composite map. With increasing satellite resolution, for example, QuickSCAT and Seawind for scatterometers (25 with $5 \mathrm{~km}$ resolution from enhanced processing), Advanced Microwave Scanning Radiometer (AMSR, $5 \mathrm{~km}$ ), and Synthetic Aperature RADAR and ScanSar (SAR and RADARSAT, $100 \mathrm{~m}$ ), it will be possible to derive sea ice motion and comparably scaled deformation products for finer temporal and spatial grids. Such results can be used to improve results found here, improve our understanding of sea ice processes, and provide a resource for numerical models.

Acknowledgments. This work was supported by funding through NASA Polar Programs, GVSP contracts NAS5-32484 and NAS-5-98181, and NSF Grant OPP9818645. Thanks also go to IABP for their buoy database available at http://iabp.apl.washington.edu. Additional thanks go to the anonymous reviewers whose comments led to improvements in the paper.

\section{References}

Beers, Y., Propagation of Error, in Introduction to the Theory of Error, Chap. 5, pp. 26-36, Addison-WesleyLongman, Reading, Mass., 1957.

Drinkwater, M.R., Active microwave remote sensing observations of Weddell sea ice, in Antarctic Sea Ice: Physical Processes, Interactions and Variability, Anarct. Res. Ser., vol. 74, pp. 187-212, edited by M.O. Jeffries, $A G U$, Washington, D.C., 1998 .

Drinkwater, M.R., Satellite microwave radar observations of Antarctic sea ice, in Analysis of SAR Data of the Polar Oceans, edited by C.Tsatsoulis and R. Kwok, pp. 145-187, Springer-Verlag, New York, $1998 b$.

Emery, W. J., C.W. Fowler, J. Hawkins, and R. H. Preller, Fram Strait satellite image-derived ice motions, J. Geophys. Res., 96(C5), 8917-8920, 1991.

Fily, M., and D. A. Rothrock, Sea ice tracking by nested correlations, IEEE Trans, Geosci. Remote Sens., GE25(5), 570-580, 1987.

Gerger, C.A., S.F. Ackley, and W.D. Hibler III, Sea ice drift and deformation processes in the western Weddell Sea, in Antarctic Sea Ice: Physical Processes, Interactions and Varnability, Anarct. Res. Ser., vol. 74, pp. 141-160, edited by M.O. Jeffries, AGU, Washington, D.C., 1998a.

Geiger, C.A., W.D. Hibler III, and S.F. Ackley, Modeled versus observed drift and deformation in the western Weddell Sea during 1992, J. Geophys. Res., 103(C10), 21893. 21914, $1998 b$.

Hibler, W. D., W. F. Weeks, A. Kovacs, and S. F. Ackley, Differential sea ıce drift, Part 1, Spatial and temporal variations in sea ice deformation, J. Glaciol., 13(69), $437-455,1974$.
Hines, W. W. and D. C. Montgomery, Multiple Regression, in Probability and Statistics in Engineering and Management Science 3rd ed., Chap. 15, pp. 487-558, John Wiley, New York, 1990.

Kwok, R., J. C. Curlander, R. McConnell, and S. Pang, An ice motion tracking system at the Alaska SAR facility, IEEE J. Oceanic Eng. 15(1), 44-54, 1990.

Kwok, R., A. Schweiger, D. A. Rothrock, S. Pang, and C. Kottmeier, Sea ice motion from from satellite passive $m i$ crowave imagery assessed with ERS SAR and buoy motions, J. Geophys. Res., 103,(C4) 8191-8214, 1998.

$L i, S ., Z$. Cheng, and W.F. Weeks, Extraction of intermediate scale sea ice deformation parameters from $S A R$ ice motion products, in Analysis of SAR Data of the Polar Oceans, Edited by C.Tsatsoulis and R. Kwok, pp. 6990,Springer-Verlag, New York, 1998.

Liu, A. K. and D. J. Cavalieri, On sea ice drift from the wavelet analysis of the Defense Meteorological Satellite Program (DMSP) Special Sensor Microwave Imager $(S S M / I)$ data, Int. J. Remote Sens., 19(7), 1415-1423, 1998.

Liu, A. K., Y. Zhao, and W. T. Liu, Sea-ice motion derived from satellite agrees with buoy observations, Eos Trans, $A G U, 79(30), 353-359,1998$.

Liu, $A$. K. and $Y$. Zhao, Sea ice motion from wavelet analysis of satellite data, paper presented in Eigth International Offshore and Polar Engineering Conference, Montreal, Canada, May 24-29, 1998.

Liu, A. K., Y. Zhao, and S. Y. Wu, Arctic sea ice druft from wavelet analysis of NSCAT and SSM/I data, J. Geophys. Res., 104(C5), 11529-11538, 1999.

Stern, H. L., D. A. Rothrock, and R. Kwok, Open water production in Arctic sea ice: Satellite measurements and model parametriation, J. Geophys. Res., 100(C10), 20601-20612, 1995.

Thorndike, A. S., Kinematics of sea ice, in The Geophysics of Sea Ice, NATO ASI Series, Series B, vol. 146, Chap. 7, pp. 489-549, edited by N. Untersteiner, Plenum, New York, 1986.

Thorndike, A. S., and R. Colony, Large-scale ice motion in the Beaufort Sea during AIDJEX, April 1975-April 1976, in Sea Ice Processes and Models, pp. 249-260, Unvv. of Wash., Seattle, 1980.

Zhao, Y., A.K. Liu, and C. A. Geiger, Arctic sea ice motion from wavelet analysis on $S S M / I$ data, in J. Adv. Mar. Sci. Tech. Soci., vol. 4, Advanced Marine Science and Technology Society, Tokyo, Japan, in press, 1999.

Cathleen A. Geiger, Center for Climatic Research, Department of Geography, 216 Pearson Hall, University of Delaware, Newark, DE 19716. (geiger@geog.udel.edu)

Sirpa M. Häkkinen and Antony K. Liu, NASA/Oceans and Ice Branch, Code 971, Goddard Space Flight Center, Greenbelt, MD 20771.

Yunhe Zhao, Caelum Research Corporation, 1700 Research Blvd., Suite 100, Rockville, MD 20850.

(Received January 15, 1999; revised July 20, 1999; accepted October 15, 1999.) 